\title{
Factores asociados a hospitalizaciones inadecuadas con protocolo de evaluación de adecuación en Instituto Especializado Pediátrico
}

\author{
Factors associated with inadequate hospitalizations with a protocol of \\ assessment of adequacy in the Specialty Institute Pediatric \\ Erika Espinoza Carrión (ORCID 0000- 0002- 2784- 5842)ํㅜ, Giovanna Huamán \\ Reyes (ORCID 0000- 0002- 0649- 1686) ${ }^{1}$, Claudia Arispe Alburqueque (ORCID \\ 0000-0003-0792-4655) ${ }^{1}$ \\ ${ }^{1}$ Universidad Privada Norbert Wiener
}

\begin{abstract}
Resumen
Las hospitalizaciones inadecuadas se realizan cuando los pacientes son internados sin una justificación válida; es decir, los cuidados dependen del estado clínico del paciente para que la atención pueda ser manejada en un nivel primario, en comparación de los pacientes que realmente ameritan una atención especializada. El objetivo del estudio fue determinar la relación entre factores asociados y hospitalizaciones inadecuadas con protocolo de evaluación de adecuación en un Instituto Especializado Pediátrico. La investigación fue cuantitativa, descriptiva y no experimental de corte transversal. La población del estudio estuvo conformada por 150 historias clínicas y, por su parte, la muestra del estudio estuvo conformada por 79 historias clínicas del servicio de medicina B de pacientes pediátricos. Para medir las variables se hicieron uso de dos fichas de recolección de datos. Los datos obtenidos fueron digitados y ordenados en el programa Excel 2016 y, posteriormente, fueron ingresados al programa SPSS, versión 24, para su respectivo análisis. Se encontró una correlación positiva entre la dimensión factores generales y hospitalizaciones inadecuadas con protocolo de evaluación de adecuación (Rho de Sperman: 0,821 $p=0,000$ ), así como también se encontró una correlación positiva muy alta entre los factores organizativos y hospitalizaciones inadecuadas con protocolo de evaluación de adecuación (Rho de Sperman: 0,837 $p=0,000$ ). Se concluye que existe una relación positiva entre los factores asociados y hospitalizaciones inadecuadas con un protocolo de evaluación de adecuación (Rho de Sperman: 0,902 $p=0,000$ ).
\end{abstract}

Palabras clave: Factores asociados, hospitalizaciones inadecuadas, pacientes. 


\begin{abstract}
Inappropriate hospitalizations occur when patients are admitted without a valid justification; that is, care depends on the clinical status of the patient so that care can be managed at a primary level, compared to patients who really deserve specialized care. The objective of the study was to determine the relationship between associated factors and inadequate hospitalizations with the adequacy evaluation protocol in a Specialized Pediatric Institute. The research was quantitative, descriptive and not cross-sectional experimental. The study population consisted of 150 medical records and the study sample consisted of 79 medical records from the B medicine service of pediatric patients. To measure the variables, two data collection cards were used. The data obtained was entered and ordered in the Excel 2016 program, and later, it entered into the SPSS program, version 24, for their respective analysis. A positive correlation between the dimension general factors and inadequate hospitalizations with the adequacy evaluation protocol (Sperman's Rho: $0.821 p=0.000$ ) was found, as well as a very high positive correlation was also found between the organizational factors and inadequate hospitalizations with the adequacy evaluation protocol (Sperman's rho: $0.837 p=0.000$ ). It is concluded that there is a positive relationship between the associated factors and inadequate hospitalizations with the adequacy evaluation protocol (Sperman's Rho: $0.902 p=0.000)$.
\end{abstract}

\title{
Keywords: Associated factors, inadequate hospitalizations, patients.
}

\section{Introducción}

Las hospitalizaciones inadecuadas (HI) tienen lugar cuando los pacientes son internados sin una justificación válida; es decir, por el estado clínico o los cuidados que requiere el paciente, este puede muy bien ser atendido fuera del hospital en menoscabo de quienes realmente necesitan ocupar camas hospitalarias $(1,2)$. Los primeros estudios sobre la utilización de los servicios sanitarios en los que se observó la pertinencia de una hospitalización se realizaron en la década de los setenta; se puede distinguir la hospitalización inadecuada, también denominada innecesaria o injustificada. Por ello, en la actualidad, esto constituye un problema central en los sistemas de salud de países desarrollados y tiene un mayor impacto en los países en vías de desarrollo, dado que eleva el gasto sanitario e incrementa la ineficiencia en la gestión, de manera tal que se influye en la calidad asistencial $(3,4)$.

Las hospitalizaciones inadecuadas a nivel mundial presentan una frecuencia que varía entre el $10 \%$ hasta el $15 \%$ del total de admisiones hospitalarias. Sin embargo, a nivel de los establecimientos pediátricos, estas pueden llegar a ser entre el $25 \%$ hasta el $45 \%$. Por otro lado, en Latinoamérica se reporta que son responsables del $12 \%$ hasta el $60 \%$ de las estancias hospitalarias prolongadas y del $6 \%$ al $54 \%$ de los ingresos hospitalarios injustificados (5, $6,7)$.

En los últimos años, el incremento de pacientes y los escasos recursos económi- 
cos disponibles han ocasionado que el indicador de eficiencia y calidad de atención en la salud hospitalaria sea el mismo porcentaje de hospitalizaciones inadecuadas $(6,7,8)$. La primera causa de hospitalizaciones inadecuadas reconocidas por los médicos es la hospitalización de los pacientes para realizar estudios especializados imprescindibles que, generalmente, se pueden hacer ambulatoriamente pero que, por el sistema de citas, se dan en plazos muy largos, lo cual no permite realizar el diagnóstico ni tratamiento a corto plazo $\mathrm{y}$, de esa manera, evitar complicaciones o secuelas prevenibles (9), con mucha frecuencia se hospitalizan a pacientes con enfermedades crónicas o con complicaciones, los mismos que podrían ser diagnosticados y tratados en consultas externas o en centros médicos de menor nivel $(10,11)$. No se tienen estadísticas sobre las hospitalizaciones inadecuadas en Institutos Pediátricos en el Perú ni tampoco se conocen los factores asociados sobre los cuales se pudieran intervenir para reducir los indicadores actuales (12).

Debido a lo anteriormente mencionado, es importante señalar que la investigación se realizó en una institución del Ministerio de Salud que está especializada en la atención de la población infantil, para ver si existe una relación entre los factores asociados y las hospitalizaciones inadecuadas con un protocolo de evaluación de la adecuación en un Instituto Especializado Pediátrico.

\section{Materiales y método}

El estudio fue realizado con un enfoque cuantitativo, de nivel descriptivo, de diseño observacional y de corte transversal. La población del estudio estuvo conformada por 150 historias clínicas del servicio de medicina $\mathrm{B}$ de pacientes pediátricos que fueron hospitalizados en un Instituto Especializado Pediátrico y la muestra del estudio estuvo conformada por 79 historias clínicas. La unidad de análisis fue la historia clínica. La técnica de muestreo fue no probabilística por conveniencia, se incluyeron las historias clínicas que contengan todos los datos solicitados, con escritura legible y que pertenezcan a pacientes pediátricos que fueron hospitalizados en el servicio de medicina B. La técnica aplicada fue de recopilación bibliográfica mediante la revisión de los datos consignados en las historias clínicas. Los instrumentos aplicados fueron dos fichas de recolección datos en donde se consignaron las variables de interés: factores asociados y hospitalizaciones inadecuadas con protocolo de evaluación de adecuación de las historias clínicas de los pacientes pediátricos que fueron hospitalizados en el servicio de medicina B.

En el análisis estadístico se obtuvieron las medidas estadísticas de frecuencia y porcentaje para la estadística descriptiva y el estadístico Rho de Sperman para conocer la correlación entre ambas variables.

Dentro de los aspectos éticos podemos describir que se contó con el permiso del hospital para realizar la investigación teniendo en cuenta que se reservó el anonimato de las historias clínicas. 
Tabla 1: Distribución de frecuencias y porcentajes de las historias clínicas de la variable 1: factores asociados

\begin{tabular}{llllll}
\hline & & Frecuencia & Porcentaje & Porcentaje válido & Porcentaje acumulado \\
\hline \multirow{4}{*}{ Válido } & Bajo & 1 & 1,3 & 1,3 & 1,3 \\
& Medio & 22 & 27,8 & 27,8 & 29,1 \\
& Alto & 56 & 70,9 & 70,9 & 100,0 \\
& Total & 79 & 100,0 & 100,0 & \\
\hline
\end{tabular}

Fuente: Base de datos

Tabla 2: Distribución de frecuencias y porcentajes de las historias clínicas de las dimensiones de la variable 1: factores asociados

\begin{tabular}{lllll}
\hline & \multicolumn{2}{c}{ Factores generales } & \multicolumn{2}{l}{ Factores organizativos } \\
\hline Niveles & fi & F $\%$ & fi & F $\%$ \\
Bajo & 8 & 10,1 & 8 & 10,1 \\
Medio & 20 & 25,3 & 17 & 21,5 \\
Alto & 51 & 64,6 & 54 & 68,4 \\
Total & 79 & 100,0 & 79 & 100,0 \\
\hline \multicolumn{4}{c}{ Fuente: Base de datos }
\end{tabular}

\section{Resultados}

Dentro de la estadística descriptiva se evidenció que las fichas de recolección de datos sobre factores asociados con una muestra de 79 historias clínicas representada por el $100 \%$ dio a conocer que el $70,9 \%$ alcanzaron niveles altos, el 27,8\% niveles medios y el 1,3\% niveles bajos, lo cual mostró que la mayoría de historias clínicas presentaron niveles altos en la variable 1: factores asociados (Tabla 1 ).

Se evidenció que, en la dimensión factores generales, las fichas de recolección de datos sobre factores asociados alcanzaron niveles alto con un el 64,6\%, el 25,3\% niveles medios y el 10,1\% niveles bajos. En la dimensión factores organizativos, el $68,4 \%$ alcanzaron niveles altos, el 21,5\% niveles medios y el 10,1\% niveles bajos, en donde prevalece los niveles altos en todas las dimensiones de la variable 1: factores asociados (Tabla 2).

Asimismo, se evidenció que las fichas de recolección de datos sobre hospitalizaciones inadecuadas con protocolo de evaluación de adecuación, con una muestra de 79 historias clínicas representada por el $100 \%$, dio a conocer que el $73,4 \%$ alcanzaron niveles medios, el 26,6\% niveles altos y ninguno niveles bajos, lo cual mostró que la mayoría de historias clínicas presentaron niveles medios en la variable 2: hospitalizaciones inadecuadas con protocolo de evaluación de adecuación (Tabla $3)$.

También se evidenció que las fichas de recolección de datos sobre hospitalizacio- 


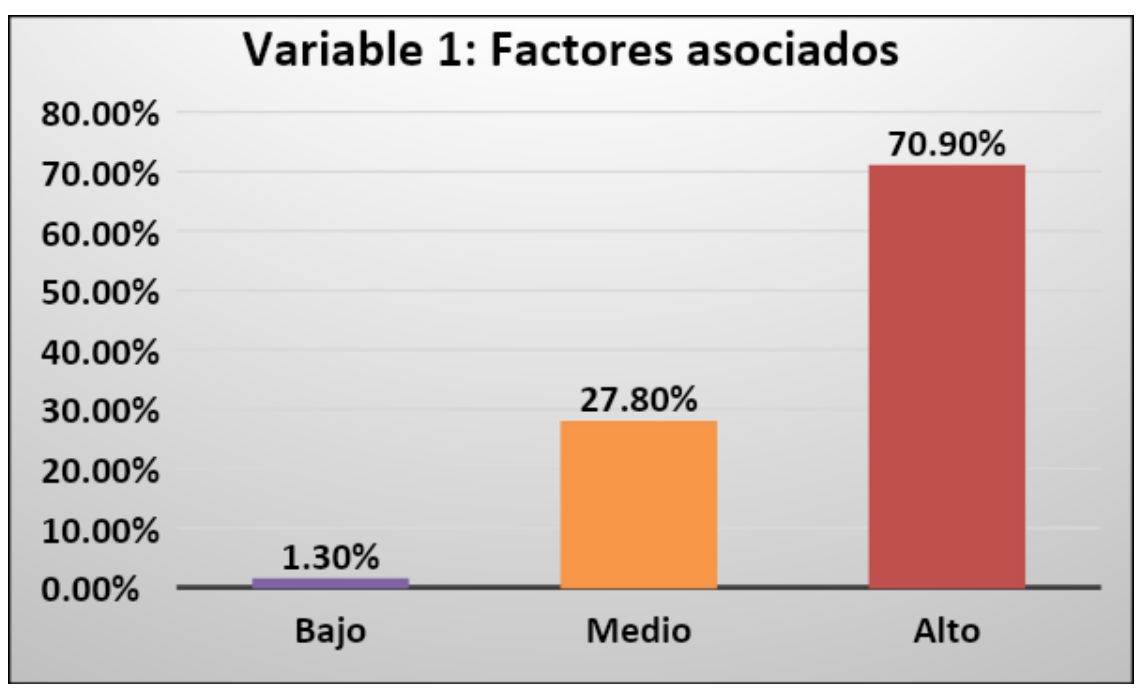

Figura 1: Distribución de frecuencias y porcentajes de las historias clínicas de la variable 1: factores asociados

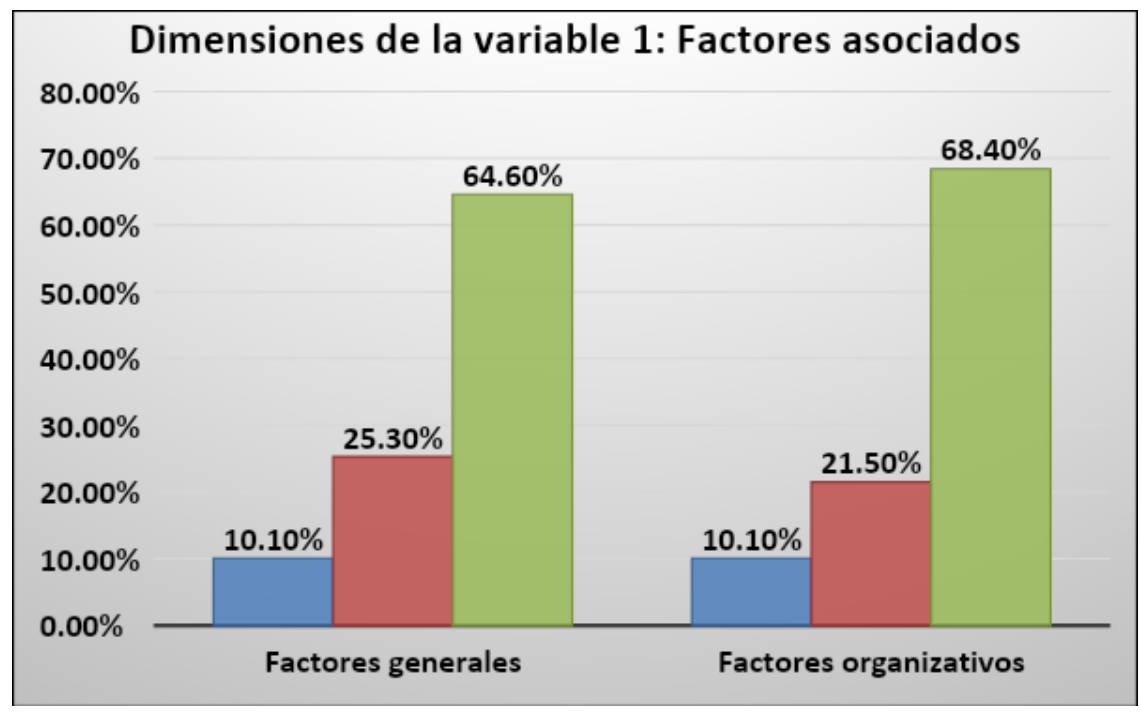

Figura 2: Distribución de frecuencias y porcentajes de las historias clínicas de las dimensiones de la variable 1: factores asociados 
Tabla 3: Distribución de frecuencias y porcentajes de las historias clínicas de la variable 2: hospitalizaciones inadecuadas con protocolo de evaluación de adecuación

\begin{tabular}{llllll}
\hline & & Frecuencia & Porcentaje & Porcentaje válido & Porcentaje acumulado \\
\hline \multirow{4}{*}{ Válido } & Bajo & - & - & - & - \\
& Medio & 58 & 73,4 & 73,4 & 73,4 \\
& Alto & 21 & 26,6 & 26,6 & 100,0 \\
& Total & 79 & 100,0 & 100,0 & \\
\hline \multicolumn{5}{c}{ Fuente: Base de datos }
\end{tabular}

Tabla 4: Distribución de frecuencias y porcentajes de las historias clínicas de las dimensiones de la variable 2: hospitalizaciones inadecuadas con protocolo de evaluación de adecuación

\begin{tabular}{lllll}
\hline & \multicolumn{2}{c}{$\begin{array}{l}\text { Criterios referentes a la situación } \\
\text { clínica del paciente }\end{array}$} & \multicolumn{2}{l}{$\begin{array}{l}\text { Criterios referentes a la intensidad } \\
\text { de cuidados prestados }\end{array}$} \\
\hline Niveles & fi & F $\%$ & fi & F \% \\
Bajo & 14 & 17,7 & 0 & 0,0 \\
Medio & 51 & 64,6 & 48 & 60,8 \\
Alto & 14 & 17,7 & 31 & 39,2 \\
Total & 79 & 100,0 & 79 & 100,0 \\
\hline
\end{tabular}

Fuente: Base de datos

nes inadecuadas con protocolo de evaluación de adecuación, en la dimensión criterios referentes a la situación clínica del paciente, alcanzaron niveles medios con un $64,6 \%$, el $17,7 \%$ niveles altos y bajos cada uno; y, en la dimensión criterios referentes a la intensidad de cuidados prestados, el $60,8 \%$ alcanzaron niveles medios, el $39,2 \%$ niveles altos y ninguno en el nivel bajo, en donde prevalece los niveles medios en todas las dimensiones de la variable 2: hospitalizaciones inadecuadas con protocolo de evaluación de adecuación (Tabla 4).

El objetivo general de la investigación fue determinar la relación entre factores asociados y hospitalizaciones inadecuadas con protocolo de evaluación de adecuación en un Instituto Especializado Pediátrico. Por ello, en la tabla 5 se visualiza que solo el 26,6\% de las historias clínicas mostraron que los factores asociados y las hospitalizaciones inadecuadas con protocolo de evaluación de adecuación tuvieron porcentajes altos. El 27,8\% de historias clínicas mostraron que los factores asociados tuvieron porcentajes medios $\mathrm{y}$, de igual forma, las hospitalizaciones inadecuadas con protocolo de evaluación de adecuación. Por último, ninguna historia clínica indicó que los factores asociados y hospitalizaciones inadecuadas con protocolo de evaluación de adecuación tuvieron porcentajes bajos. Por lo tanto, de acuerdo a los resultados obtenidos se puede afirmar que los factores asociados tuvieron 


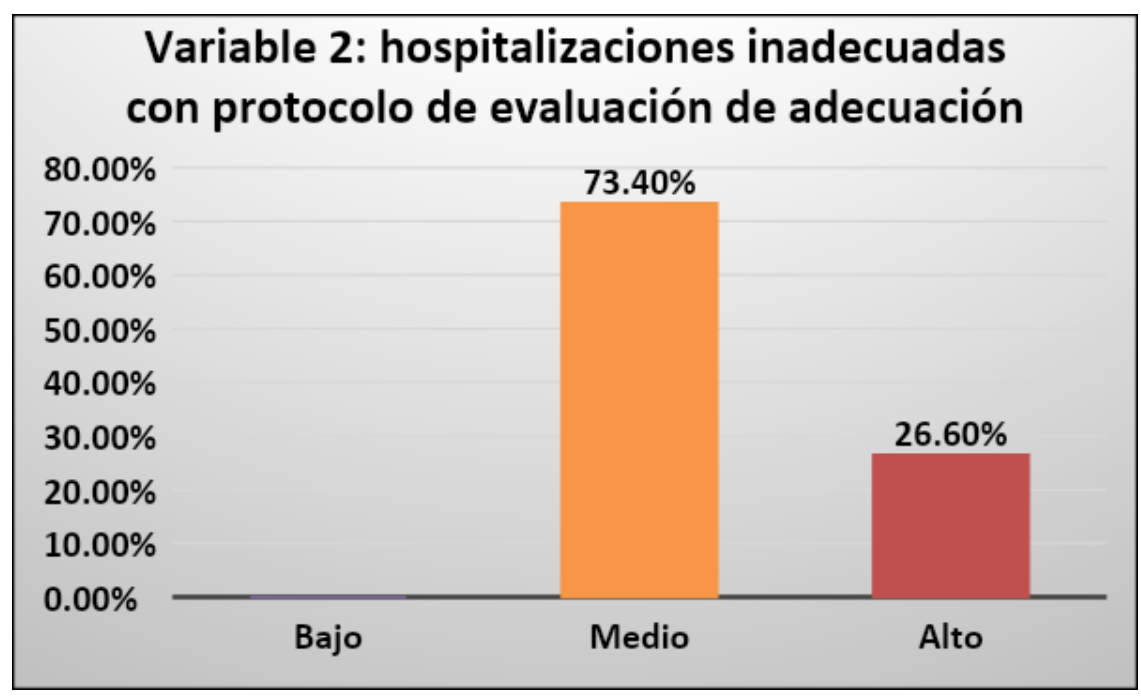

Figura 3: Distribución de frecuencias y porcentajes de las historias clínicas de la variable 2: hospitalizaciones inadecuadas con protocolo de evaluación de adecuación

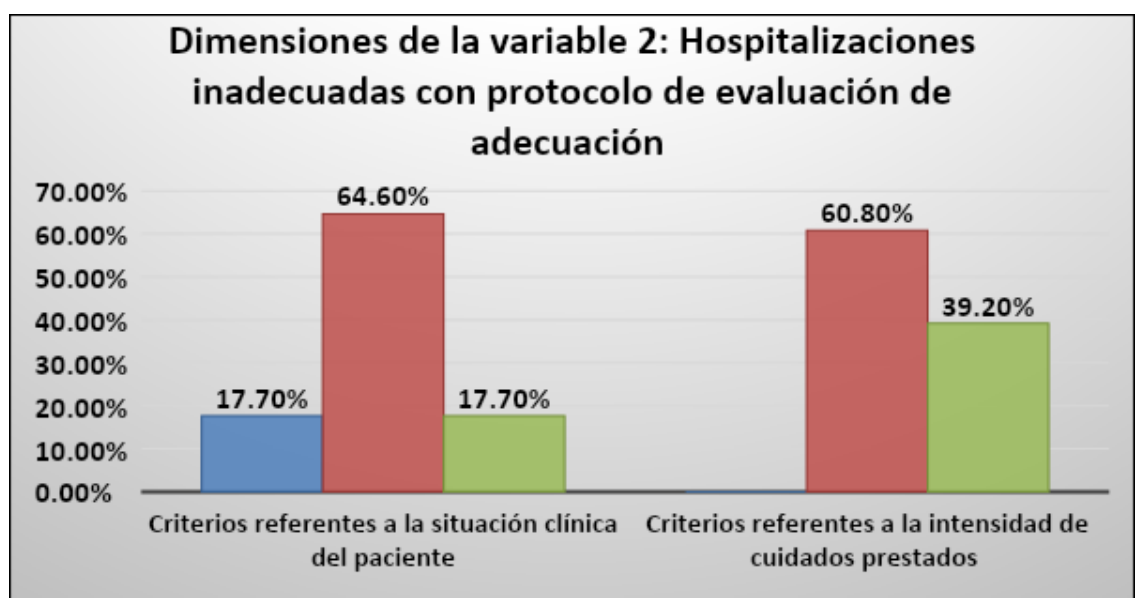

Figura 4: Distribución porcentual de las historias clínicas de las dimensiones de la variable 2: hospitalizaciones inadecuadas con protocolo de evaluación de adecuación 
Tabla 5: Tabla de contingencia: factores asociados y hospitalizaciones inadecuadas con protocolo de evaluación de adecuación

\begin{tabular}{llllllllllll}
\hline & \multicolumn{8}{c}{ Hospitalizaciones inadecuadas con protocolo de } \\
evaluación de adecuación
\end{tabular}

Tabla 6: Prueba de Spearman de la correlación de las variables factores asociados y hospitalizaciones inadecuadas con protocolo de evaluación de adecuación

\begin{tabular}{lll}
\hline & & $\begin{array}{l}\text { Hospitalizaciones inadecuadas con protocolo de } \\
\text { evaluación de adecuación }\end{array}$ \\
\hline \multirow{3}{*}{ Factores asociados } & Correlación de Spearman &, $902^{* *}$ \\
& Sig. (bilateral) &, 000 \\
& $\mathrm{~N}$ & 79 \\
\hline
\end{tabular}

* La correlación es significativa en el nivel 0,01 (bilateral). Fuente: Programa SPSS v.24

porcentajes altos en un 70,9\%, y las hospitalizaciones inadecuadas con protocolo de evaluación de adecuación tuvieron porcentajes medios en un $73,4 \%$ (Tabla 5).

Dentro de la estadística inferencial encontramos que los factores asociados y hospitalizaciones inadecuadas con protocolo de evaluación de adecuación se relacionan positivamente, dado que según el coeficiente Rho de Spearman, 0,902 las variables muestran una correlación positiva muy alta (Tabla 6). Dentro de los factores asociados, según la dimensión factores generales y hospitalizaciones inadecuadas con protocolo de evaluación de adecuación, se relacionan positivamente, ya que según el coeficiente Rho de Spearman,
0,821 de las variables muestran una correlación positiva muy alta (Tabla 7 ).

Finalmente, los factores organizativos y hospitalizaciones inadecuadas con protocolo de evaluación de adecuación se relacionan positivamente, pues, según el coeficiente Rho de Spearman, 0,837 las variables muestran una correlación positiva muy alta (Tabla 8).

\section{Discusión}

Debido al considerable incremento de pacientes y los escasos recursos económicos disponibles para brindar una atención especializada, racional y pertinente 
Tabla 7: Prueba de Spearman de la correlación de las variables factores asociados, según la dimensión factores generales, y hospitalizaciones inadecuadas con protocolo de evaluación de adecuación

\begin{tabular}{lll}
\hline & & $\begin{array}{l}\text { Hospitalizaciones inadecuadas con protocolo de } \\
\text { evaluación de adecuación }\end{array}$ \\
\hline \multirow{3}{*}{ Factores generales } & Correlación de Spearman &, $821^{* *}$ \\
& Sig. (bilateral) &, 000 \\
& $\mathrm{~N}$ & 79 \\
\hline
\end{tabular}

* La correlación es significativa en el nivel 0,01 (bilateral). Fuente: Programa SPSS v.24

Tabla 8: Prueba de Spearman de la correlación de las variables factores asociados, según la dimensión factores organizativos, y hospitalizaciones inadecuadas con protocolo de evaluación de adecuación

\begin{tabular}{lll}
\hline & & $\begin{array}{l}\text { Hospitalizaciones inadecuadas con protocolo de } \\
\text { evaluación de adecuación }\end{array}$ \\
\hline \multirow{3}{*}{ Factores organizativos } & Correlación de Spearman &, $837^{* *}$ \\
& Sig. (bilateral) &, 000 \\
& $\mathrm{~N}$ & 79 \\
\hline
\end{tabular}

** La correlación es significativa en el nivel 0,01 (bilateral). Fuente: Programa SPSS v.24

en cuanto a las camas hospitalarias, el indicador de eficiencia y calidad de atención en la salud hospitalaria se ve afectada por las hospitalizaciones inadecuadas que inciden en los recursos económicos, recursos humanos, recursos tecnológicos y los recursos que tienen que ver con la infraestructura hospitalaria, ya que estos son los factores organizativos que se encuentran asociados a hospitalizaciones inadecuadas con protocolo de evaluación de la adecuación en Instituto Especializado Pediátrico, los cuales son necesarios adecuar para realizar acciones de intervención y disminuir las tasas actuales $(1,2,13,14)$.

De acuerdo con estos resultados, es posible señalar que los factores asociados se relacionan con las hospitalizaciones inade- cuadas con protocolo de evaluación de adecuación lo que concuerda con lo expresado por Contreras (15), quien mencionó en su investigación que las hospitalizaciones inadecuadas (innecesarias, injustificadas) son realizadas en hospitales de gran complejidad que generan ingresos innecesarios, aunque, en realidad, podrían haberse realizado en niveles asistenciales distintos: ambulatorio, consulta domiciliaria, hospital de cuidados crónicos o en centros de menor nivel de complejidad. Esto asume que los cuidados prestados a los pacientes son siempre pertinentes desde el punto de vista clínico y son diversos los factores asociados que pueden influenciar en la hospitalización inadecuada en los servicios de medicina, desde la condi- 
ción social del paciente, edad del paciente, hasta el familiar que lo trae o acompaña. Esto difiere de la investigación de Chirinos (16), quien indicó que los factores asociados a las hospitalizaciones inadecuadas fueron las patologías crónicas y de hospitalizaciones anteriores del niño, quienes ingresaron para esperar una prueba diagnóstica, la admisión por el área de emergencia, peso al nacer y no provenir con referencia. Además, sumado a lo anterior, se ratifica lo que expresaron $\mathrm{Ne}$ koei, Amiresmaili, Goudarzi et al (17) en sus estudios sobre la idoneidad de la admisión y hospitalización en un hospital, ya que solo se deben realizar hospitalizaciones adecuadas a pacientes que necesiten tratamientos médicos, de enfermería y paramédicos continuos y activos, y no se puede realizar la prestación de estos servicios en otros lugares como centros de día y ambulatorios.

Asimismo, en los resultados de este estudio se observó que los factores asociados, según la dimensión factores generales, y hospitalizaciones inadecuadas con protocolo de evaluación de adecuación se relacionan positivamente en un Instituto Especializado Pediátrico. Así es posible señalar que la primera dimensión de la variable factores generales se relaciona con la variable hospitalizaciones inadecuadas con protocolo de evaluación de adecuación, lo cual es similar a lo expresado por Valentín (18). Valentín mencionó en su investigación que las hospitalizaciones inadecuadas o innecesarias resultan de varios factores además de los generales, tales como la saturación de los procesos de atención en la consulta externa, hospitales carentes de varias de las pruebas comple- mentarias necesarias para un diagnóstico precoz y rápido, establecimientos de salud de primer nivel de atención que no resuelven los problemas de salud de la población ocasionando malestar y generando que prefieran acudir primero al hospital antes que al puesto de salud, sistemas desordenados y bidireccionados de salud, entre otros, lo cual difiere de las investigaciones de Nekoei, Amiresmaili, Goudarzi, et al (17). Estos indicaron que los factores generales del paciente no es lo más importante, sino que, si las medidas estándar de admisión y hospitalización se ejecutan correctamente, se puede reducir la tasa de hospitalización innecesaria, de modo que se pueda tratar a más pacientes y se modere el costo y la carga de trabajo del personal del hospital.

Además, se confirma lo que sostiene Chirinos (16) en sus estudios sobre admisión hospitalaria en pediatría, quien, al realizar el análisis de la relación entre las características clínicas y la admisión hospitalaria, encontró una significancia estadística en los siguientes factores: edad, el distrito de procedencia alejado del hospital, el ingreso por emergencia, ser hospitalizado por especialidad Pediatría, admisión hospitalaria en turno tarde, tiempo de enfermedad crónico y antecedente de hospitalización anterior y antecedente quirúrgico.

Finalmente, de acuerdo con los resultados del estudio se observó que los factores asociados según la dimensión factores organizativos y hospitalizaciones inadecuadas con el protocolo de evaluación de adecuación se relacionan positivamente en un Instituto Especializado Pediátrico. Por ello, de acuerdo con estos resultados es po- 
sible señalar que la segunda dimensión de la variable factores organizativos se relaciona con las hospitalizaciones inadecuadas con protocolo de evaluación de adecuación, lo cual es similar expresado por Valentín (18). Este mencionó en su investigación que la relevancia de realizar una conveniente hospitalización inadecuada implica costos innecesarios a la familia del paciente y al establecimiento de salud; asimismo, puede llegar a exponer al paciente a riesgos como infecciones nosocomiales, procedimientos dolorosos, incidentes con el personal de salud y en el caso especial de la población pediátrica, a periodos de tensión, y se constituye como la sexta causa de estrés más importante asociada a cambios en el comportamiento y en la salud mental, además de todos los factores organizativos que se relacionan con las hospitalizaciones inadecuadas. Esto difiere de la investigación de Chirinos (16), quien indicó que se debe de dar prioridad a los pacientes con mayor compromiso clínico para la toma de exámenes diagnósticos y desarrollo de procedimientos sin considerar donde se encuentren estos -hospitalizados, emergencia, consulta externa, entre otros- y no solo a los factores organizativos como la especialidad del médico tratante o turno de admisión.

En adición, se ratifica lo que expresaron Vado y Sánchez (19) en sus estudios sobre la evolución de la adecuación de las hospitalizaciones en pacientes pediátricos, puesto que estos investigadores notaron que cualquier rasgo, característica o peculiaridad provoca una hospitalización inadecuada, ya sea un ingreso innecesario o una estancia prolongada, además de los factores generales y organi- zativos que influyen en las hospitalizaciones inadecuadas.

Reconocer los factores asociados a hospitalizaciones inadecuadas podría ser de gran aporte a futuras investigaciones, ya que se pueden establecer protocolos de control ante una hospitalización previa, con lo cual se disminuiría el gasto hospitalario. Dentro de las limitaciones para realizar la investigación podemos contar a la dificultad para obtener el total de las historias clínicas y la calidad de las mismas por ausencia de datos o escritura ilegible.

\section{Conclusiones}

A partir del estudio realizado, se puede concluir que los factores asociados y hospitalizaciones inadecuadas con protocolo de evaluación de adecuación se relacionan positivamente en un Instituto Pediátrico Especializado.

\section{Referencias}

(1) Castellanos A, Cuellar H. Causales de estancias prolongadas de pacientes hospitalizados en las unidades de cuidados intensivos. [Tesis para optar el título de especialista en auditoria en Salud]. Colombia: Universidad Santiago de Cali, 2020. Disponible en: https://repository.usc. edu.co/bitstream/handle/20.500. 12421/5072/CAUSALES\%20DE\% 20ESTANCIA.pdf? sequence $=3 \&$ isAllowed $=\mathrm{y}$

(2) Moscoso N.S, Pisani M.V. Internaciones inadecuadas: su impacto en la 
eficiencia del uso de la cama hospitalaria. Revista de ciencias sociales. 2017; 23(3):39-47. https://doi.org/ $10.31876 /$ rcs.v23i3.25128

(3) Ghods A, Khabiri R, Raisdana N, Ansari M, Motlagh N, Sadeghi, M. et al. Predictors of Inappropriate Hospital Stay: Experience from Iran. Journal Glob Health. 2010; $7(3): 82-89$.

(4) Menand E, Lenain E, Lazarovici C, Chatellier G, Saint-Jean O, Somme D y et al. French Multicenter Evaluation of the Appropriateness of Admission to the Emergency Department of the Over -80 s. Journal Nutr Health Aging. 2015; 19(6):681687.

(5) López P, García N, Cubillana H, Gómez C y Cánovas G. Estratificación y monitorización de niveles de adecuación de ingresos urgentes en un hospital de tercer nivel. Journal Calid Asist. 2014; 29(1):10-16.

(6) Fekari J, Ghiasi A, Ezzati M, et al. The Assessing of Inappropriate Admissions and Hospitalization based on Appropriate Evaluation Protocol in Alinasab hospital in Tabriz-2009, Journal of Hospital (JHOSP). 2011; 9(4):39-44. http://jhosp.tums.ac.ir/ article-1-77-en.html

(7) Soria V, Carrillo A, Campillo A, et al. Associated factors and cost of inappropriate hospital admissions and stays in a second-level hospital, Am J Med Qual. 2009; 24(2):321332 .
(8) Raile A, Marrier T. Modelos y Teorías de Enfermería. $7^{\circ}$ Ed. El Sevier: España. 2011.

(9) Ayuso M, Grande S. La Gestión de Enfermería y los Servicios Generales en las Organizaciones Sanitarias. Editorial Díaz de Santos S.A.: Madrid; 2012. ISBN Ebook.

(10) Moscoso N.S, Pisani M.V. Internaciones inadecuadas: su impacto en la eficiencia del uso de la cama hospitalaria. Revista de ciencias sociales, 2017; 23(3):39-47. https://doi.org/ $10.31876 /$ rcs.v23i3.25128

(11) Velásquez KJV, Flores RCS. Evaluación de la adecuación de las hospitalizaciones en pacientes atendidos en el servicio de pediatría del Hospital Alemán Nicaragüense en base al Protocolo de Evaluación de la Adecuación versión pediátrica, durante el mes de febrero del 2016. [Tesis de titulación]. Nicaragua: Universidad Nacional Autónoma de Nicaragua, 2016. http://repositorio. unan.edu.ni/id/eprint/3632

(12) Bilkis M. Adecuación de los criterios de internación en hospitales pediátricos. Salud y ciencia. 2018; 22(8):772-77.

(13) Tao J, Luo C, Ma J. Agreement of appropriateness evaluation protocol of admission and hospital days in department of gynecology and department of neurology, Journal of Shanghai Jiaotong University (Medical Science). 2013; 24(6):21-31. 
(14) Fontaine P, Jacques J, Gillain D, et al. Assessing the causes inducing lengthening of hospital stays by means of the Appropriateness Evaluation Protocol, Health Policy. 2011; 99(1):66-71. ne, P.; Jacques, J.; Gillain, D.; Sermeus, W.; Kolh, P. y Gillet, P. Assessing the causes inducing lengthening of hospital stays by means of the Appropriateness Evaluation Protocol. Health Policy. 2011; 99(1): 66-71.

(15) Contreras C, Galarza M. Hospitalizaciones inadecuadas en emergencias médicas, Rev. Soc. Perú Medicina Interna. 2014; 24(1):25-34.

(16) Chirinos J. Admisión hospitalaria en pediatría: características clínico epidemiológicas y factores relacionados a hospitalización inadecuada en el Hospital Nacional Carlos Alberto Seguín Escobedo. [Tesis de titulación]. Perú: Universidad Nacional de San Agustín de Arequipa; 2019. http://repositorio. unsa.edu.pe/handle/UNSA/8228

(17) Nekoei M, Amiresmaili M, Goudarzi $\mathrm{R}$, et al. Investigating the Appropriateness of Admission and Hospitalization at a Teaching Hospital: A Case of a Developing Country, Iran J Public Health. 2017; 46(12):1720-1725. https://www.sid.ir/en/journal/

ViewPaper.aspx?id=610812

(18) Valentín, B. Hospitalizaciones inadecuadas en el servicio de oncología médica del hospital
Docente Alcides Carrión de Huancayo. [Tesis de especialidad en oncología médica]. Perú: Escuela de Postgrado Universidad Peruana Los Andes. 2018. https: //hdl.handle.net/20.500.12848/576

(19) Vado K, Sánchez R. Evaluación de la adecuación de las hospitalizaciones en pacientes atendidos en el servicio de pediatría del Hospital Alemán Nicaragüense en base al Protocolo de Evaluación de la Adecuación versión pediátrica, durante el mes de febrero del 2016. [Tesis de titulación]. Nicaragua: Universidad Nacional Autónoma de Nicaragua. 2016. http://repositorio.unan. edu.ni/id/eprint/3632 